\title{
The Effectiveness of Ketamine Compared to Opioid Analgesics for Management of Acute Pain in Children in the Emergency Department: Systematic Review
}

\author{
Elham Alenzi \\ Ministry of Health, Maternity and Child Hospital, Skaka, Saudi Arabia \\ Email: sk662@hotmail.com
}

How to cite this paper: Alenzi, E. (2021) The Effectiveness of Ketamine Compared to Opioid Analgesics for Management of Acute Pain in Children in the Emergency Department: Systematic Review. Health, 13, 1346-1364.

https://doi.org/10.4236/health.2021.1311098

Received: October 20, 2021

Accepted: November 26, 2021

Published: November 29, 2021

Copyright $\odot 2021$ by author(s) and Scientific Research Publishing Inc. This work is licensed under the Creative Commons Attribution International License (CC BY 4.0).

http://creativecommons.org/licenses/by/4.0/

\section{(c) (i) Open Access}

\begin{abstract}
Background: Ketamine is increasingly being used as an alternative to opioids in the management of acute pain in the emergency department. In turn, there is increasing research attention to prove the efficacy of ketamine as an analgesic in children presenting in the emergency department. Objective: The first objective of this systematic review was to investigate the effectiveness of ketamine compared to opioid analgesics for pain management in children aged two months to 18 years who have acute pain in the emergency department. The second objective was to compare the adverse events and side effects associated with ketamine with those associated with opioids used for pain management. Methods: A systematic review, using the JBI systematic review was completed. A computerised search from five databases; CINAHL, EMBASE, EMCARE and PubMed, and Cochrane. The included studies were appraised by JBI critical appraisal tool for randomised controlled trials and the study results analysed. Findings: Four randomised control trial studies were included in this systematic review. All the included studies compared ketamine with opioids (morphine and fentanyl) for the management of severe pain in children. The studies were of high methodological quality based on JBI critical appraisal outcome. Meta-analysis was not possible because of the heterogeneity of the studies, especially in terms of different outcome measures, and the approaches (pain assessment tool) used to measure the pain outcomes. The review identified that ketamine demonstrated a non-inferior analgesia effect compared to opioid medication (morphine or fentanyl) as determined by various pain scores used in different studies. However, ketamine use was associated with increased frequency of occurrence of temporary ad-
\end{abstract}


verse effects that do not require clinical attention. Conclusion: Based on the findings from the review, ketamine is a suitable alternative for opioid analgesics for the management of acute and severe pain in children in ED. The minor transient side effects associated with ketamine should not limit the use of ketamine. Future studies should investigate the appropriate dosage and route of administration of ketamine to be used while managing pain among children with acute and severe pain in the emergency department.

\section{Keywords}

Children, Ketamine, Opioid, Pain, Paediatrics, Intramuscular, Intranasal, Intravenous, Morphine, Management, Analgesia

\section{Introduction}

Ketamine is increasingly becoming an important alternative analgesic to opioids in managing acute and severe pain, especially in children in the emergency department [1]. Existing evidence indicates that a low dose of ketamine is as effective as more commonly used strong opioid analgesics, such as fentanyl and morphine, in the management of severe acute pain [2] [3] [4]. There have been several randomised control trials to compare ketamine and opioids for pain management [2] [3] [4]. For instance, a randomised control trial (RCT) conducted by Frey [1] involving 90 children ( 8 - 17 years) with traumatic extremity injuries identified that ketamine was comparable to fentanyl for pain alleviation, although it resulted in minor and transient adverse events [1]. Lubega [3] also compared $1 \mathrm{mg} / \mathrm{kg}$ of intravenous (I.V.) ketamine with I.V. morphine $0.1 \mathrm{mg} / \mathrm{kg}$ in managing severe pain related to sickle cell disease, and revealed that ketamine had similar analgesic effects to morphine but resulted in an increased occurrence of temporary and non-life threatening mild side effects. In view of the increased research attention on ketamine in the management of severe pain in children, this systematic review aims to consolidate available evidence on its use in managing acute and severe pain in children.

\section{Methods}

\subsection{Study Design}

First, this study was a systematic review. The design was pertinent for gathering and appraisal of evidence presented in the available RCTs regarding the use of ketamine in pain management compared to opioids among children with severe pain in ED. The systematic review design was conducted based on the protocols outlined by the Joanna Briggs Institute [5]. The Joanna Briggs Institute (2017) systematic review protocol outlines a robust approach for conducting a systematic review, which includes; formulation of a research question using the $\mathrm{P}$ (Population), I (Intervention), C (Comparison) and O (Outcome) format, conduct of a systematic literature search, development of appropriate inclusion and 
exclusion criteria and methods for quality appraisal, data extraction and analysis and synthesis of results, and finally reporting of the results.

\subsection{Data Sources}

The search for the studies included in this systematic review was conducted using a computerised search of five electronic online bibliographic databases, (CINAHL, EMBASE, EMCARE and PubMed, and Cochrane). The search of the databases was conducted on 14th May 2019.

\subsection{Search Strategy}

Key search terms and phrases to search for relevant studies from the selected databases and were derived from the PICO study question. The terms included "ketamine", "children", "paediatric", “opioids", "pain management", "acute pain", "effectiveness", “efficacy", “sedation", “analgesic" and "adverse effects" (see Table 1) for full details of the search. To narrow the scope of the search, Boolean operators, such as “AND”, “OR”, and “*”, were used to generate various search syntax (see Table 1) [6]. This yielded search phrases, such as "opioid OR opioid analgesic OR Morphine OR fentanyl” (see Table 1).

\subsection{Eligibility Criteria}

Inclusion Criteria: Randomised control trials (RCTs), experimental, and quasi-experimental studies addressing the research question were included in this review. Randomised control trial studies and experimental studies often present considerably high level of evidence. All studies that met inclusion criteria were considered for review irrespective of their publication dates. Included studies were published in the English language. Studies that involved a population of children aged one month and 18 years with severe acute pain related to trauma or other illnesses, such as sickle cell were included. This included all children presenting in ED and other care centres that provided care for children with severe acute pain. This systematic review included studies that compared the effectiveness of ketamine with strong opioids, such as morphine and fentanyl for pain management measured using different method or tools (subjective and objective pain scores), such as behavioural pain scale, visual pain scale, and or self-report

Table 1. Search terms and phrases.

\begin{tabular}{|c|c|c|c|c|c|c|c|}
\hline \multicolumn{2}{|c|}{ Population } & \multirow[b]{2}{*}{ AND } & \multirow{2}{*}{$\begin{array}{c}\text { Interventions } \\
\text { Ketamine }\end{array}$} & \multirow{2}{*}{\multicolumn{2}{|c|}{$\begin{array}{cc} & \text { Comparison } \\
\text { D } & \text { Opioid }\end{array}$}} & \multirow[b]{2}{*}{ AND } & Outcome \\
\hline Key terms & Children & & & & & & Pain \\
\hline $\begin{array}{l}\text { Synonyms } \\
\text { and related } \\
\text { search terms }\end{array}$ & $\begin{array}{l}\text { Paediatrics, } \\
\text { OR babies } \\
\text { OR Infants }\end{array}$ & & $\begin{array}{c}\text { Intramuscular } \\
\text { ketamine, OR } \\
\text { intranasal ketamine, } \\
\text { OR oral ketamine, } \\
\text { OR Intravenous ketamine }\end{array}$ & & $\begin{array}{c}\text { Morphine, OR } \\
\text { fentanyl, OR } \\
\text { Narcotics }\end{array}$ & & $\begin{array}{c}\text { Pain management OR } \\
\text { Analgesic effect OR } \\
\text { Analgesia, OR sedation, OR } \\
\text { Effectiveness, OR efficacy, } \\
\text { OR adverse effects, OR } \\
\text { Acute pain, OR Severe pain }\end{array}$ \\
\hline
\end{tabular}


pain scale. Exclusion Criteria: Non-experimental studies, case studies, review reports, and qualitative studies were excluded from the review. Studies were excluded from this systematic review if they did not included children of if they did not separate pooling and analysis of the data from neonates, adults, as well as the population of children with two or more chronic conditions and disabilities that required complex additional pain management interventions that might influence the outcome of opioids or ketamine use. Studies that did not include pain as one of the primary outcomes were excluded.

\subsection{Study Selection and Reporting}

The primary researcher (EA) screened the citations derived from the search results for eligibility by considering their titles and abstracts. A subset (20\%) was also screened by one of the two supervisors (DC or JT). The screening process involved confirmation if the studies were RCT, true-experimental, or quasi-experimental designs. Furthermore, the reviewers confirmed whether the studies met the inclusion criteria: had children as the population, ketamine use as the interventions, opioid drugs as the comparator, and pain management outcome as the primary outcome. Two of three reviewers (EA and one of either DC or JT) performed full-text screening to determine whether the articles met the inclusion criteria. The studies where eligibility was unclear were reviewed in full. All reference lists of the included studies were also assessed for any additional relevant studies (published or unpublished). For reporting of the findings of the search and eligibility screening, Preferred Reporting Items for Systematic Reviews and Meta-Analyses (PRISMA) was used.

\subsection{Critical Appraisal}

The Joanna Brigs Institute's critical appraisal tool was used to guide the assessment of the methodological quality of the included studies (Table 2) [5]. The JBI critical appraisal tool was used to establish the degree to which the included

Table 2. Crtical appraisal outcome for included studies.

\begin{tabular}{cccccccccccccc}
\hline Author and years & Q1 & Q2 & Q3 & Q4 & Q5 & Q6 & Q7 & Q8 & Q9 & Q10 & Q11 & Q12 & Q13 \\
\hline Barcelos et al. (2015) [9] & YES & YES & YES & YES & NO & NO & YES & YES & YES & YES & YES & YES & YES \\
Reynolds et al. (2017) [10] & YES & YES & YES & YES & NO & YES & YES & YES & YES & YES & YES & YES & YES \\
Lubega et al. (2018) [3] & Unclear & YES & YES & YES & YES & Unclear & YES & YES & YES & YES & Unclear & YES & YES \\
Frey et al. (2018) [1] & YES & YES & YES & YES & YES & YES & YES & YES & YES & YES & YES & YES & YES \\
\hline
\end{tabular}

Q1. Was true randomisation used for the assignment of participants to treatment groups? Q2. Was allocation to treatment groups concealed? Q3. Were treatment groups similar at the baseline? Q4. Were participants blind to treatment assignment? Q5. Were those delivering treatment blind to treatment assignments? Q6. Were outcomes assessors blind to treatment assignment? Q7. Were treatment groups treated identically other than the intervention of interest? Q8. Was follow up complete and if not, were differences between groups in terms of their follow up adequately described and analysed? Q9. Were participants analysed in the groups to which they were randomised? Q10. Were outcomes measured in the same way for treatment groups? Q11. Were outcomes measured in a reliable way? Q12. Was an appropriate statistical analysis used? Q13. Was the trial design appropriate, and any deviations from the standard RCT design (individual randomisation, parallel groups) accounted for in the conduct and analysis of the trial? 
studies endeavoured to minimise the possibility of biases related to participants' selection, randomisation, treatment allocation and blinding [7].

\subsection{Assessment of Methodological Quality}

Two of the three reviewers performed the critical appraisal of the included studies independently using the RCT critical appraisal tool provided by JBI (2017). The reviewers used the outcome to determine the internal validity and risk of bias of the included studies [7]. Then, two of the reviewers met to discuss the results and outcomes of their critical appraisal for a final appraisal. They agreed on the final critical appraisal outcome. In cases consensus was not reached, the third neutral reviewer was invited to make the final decision regarding the inclusion of the article.

\subsection{Minimisation of Bias in the Systematic Review}

To minimise potential biases in this systematic review, three reviewers were involved. The inclusion of three reviewers minimised potential biases that could have arisen from one reviewer [5]. The three reviewers used the Critical Appraisal tool provided by the JBI (2017). This helped in assessing, identifying, and reporting the risk of bias in each study [7]. Additionally, it assisted the reviewers to identify various indicators of internal validity, such as participant's allocation and concealment, researchers blinding, randomisation, equal treatments (intervention), loss of follow-up information, intention to treat, as well as subjective and objective measures with blinded ratters [5] [7].

\subsection{Data Extraction and Management}

A data extraction table provided by JBI (2017) was used to support the consistency of the data extraction process. The table enables the systematic extraction of data from the included studies. The two of the three reviewers conducted the data extraction process. The data extracted included authors of the study articles, year of publication, setting, the populations, interventions, study result, and review conclusion.

\subsection{Data Analysis Method}

A meta-analysis of the studies was intended but could not be performed because of the heterogeneity of the studies in terms of sample, setting of the study, rout of treatment administration, and outcome measurement methods. Therefore, the data was analysed and presented as a narrative analysis, which allowed the reviewers to pool the content and themes derived from the included studies. The analysis involved aggregating and synthesising findings to derive a set of statements through assembling and categorising findings based on their similarities in meaning and outcomes [5]. The category descriptions were created to derive the themes, which were identified mainly from the studies' result sections. The categories were then subjected to synthesis to produce a single comprehensive set of synthesised findings. Narrative forms were used. This involved generations 
of a set of statements that represented aggregations of key themes derived from the studies' results.

\subsection{Storage and Management of Data}

EndNote X9 2018 for the Windows operating system was used to store and manage the results of the search and the eligible studies. This tool allowed for removal of duplicate records, finding full-text articles, and creating group sets for the databases searched, the reviewers, as well as the exclusion and inclusion criteria. It was also used for annotating the record of the included study articles.

\subsection{Ethical Considerations}

Since this was a systematic review that involved pooling and appraisal of secondary data it did not generate any ethical concerns. However, the reviewer identified that all included studies reported receipt of ethics committee approval for protocol prior to conducting the study, although this was not an inclusion of exclusion criteria [8]. The reviewers declared no conflict of interest during this systematic review.

\section{Result}

\subsection{Search Strategy Outcomes and Study Inclusion}

The electronic search was performed using five databases (CINAHL, EMBASE, EMCARE and PubMed, and Cochrane) on $14^{\text {th }}$ May 2019, which yielded a total of 242 citations (see Figure 1). Once duplicates were removed and full-texts reviewed, only four studies met the inclusion and exclusion criteria and were included in the review (see Figure 1). The first and second steps of identifying the citations and retrieval and screening of abstracts were done by the primary reviewer. However, two other independent reviewers assessed and reviewed twenty percent of the abstracts and full texts of the studies. The primary reviewer screened all the identified citations and eliminated a total of 78 duplicate studies. The primary reviewer retrieved and screened 164 abstracts. One hundred and thirty-five were excluded because they were not experimental. Only 29 full-text studies were retrieved and screened for inclusion and exclusion criteria and of these 25 were excluded as they did not meet the inclusion criteria. Eleven of them recruited adults. Five studies had ketamine combined with other drugs as the intervention; one compared two routes of ketamine administration (intravenous and subcutaneous). Two studies did not have pain as a primary or secondary outcome, six had compared ketamine efficacy with other analgesics, such as NSAIDs and anaesthetics. Therefore, only four of the studies were considered suitable for inclusion in this systematic review.

\subsection{The Outcome of the Methodological Quality Assessment and Risk of Bias}

\subsubsection{Methodological Quality Assessment}

The Joanna Briggs Institute (JBI) critical appraisal tool for randomised 

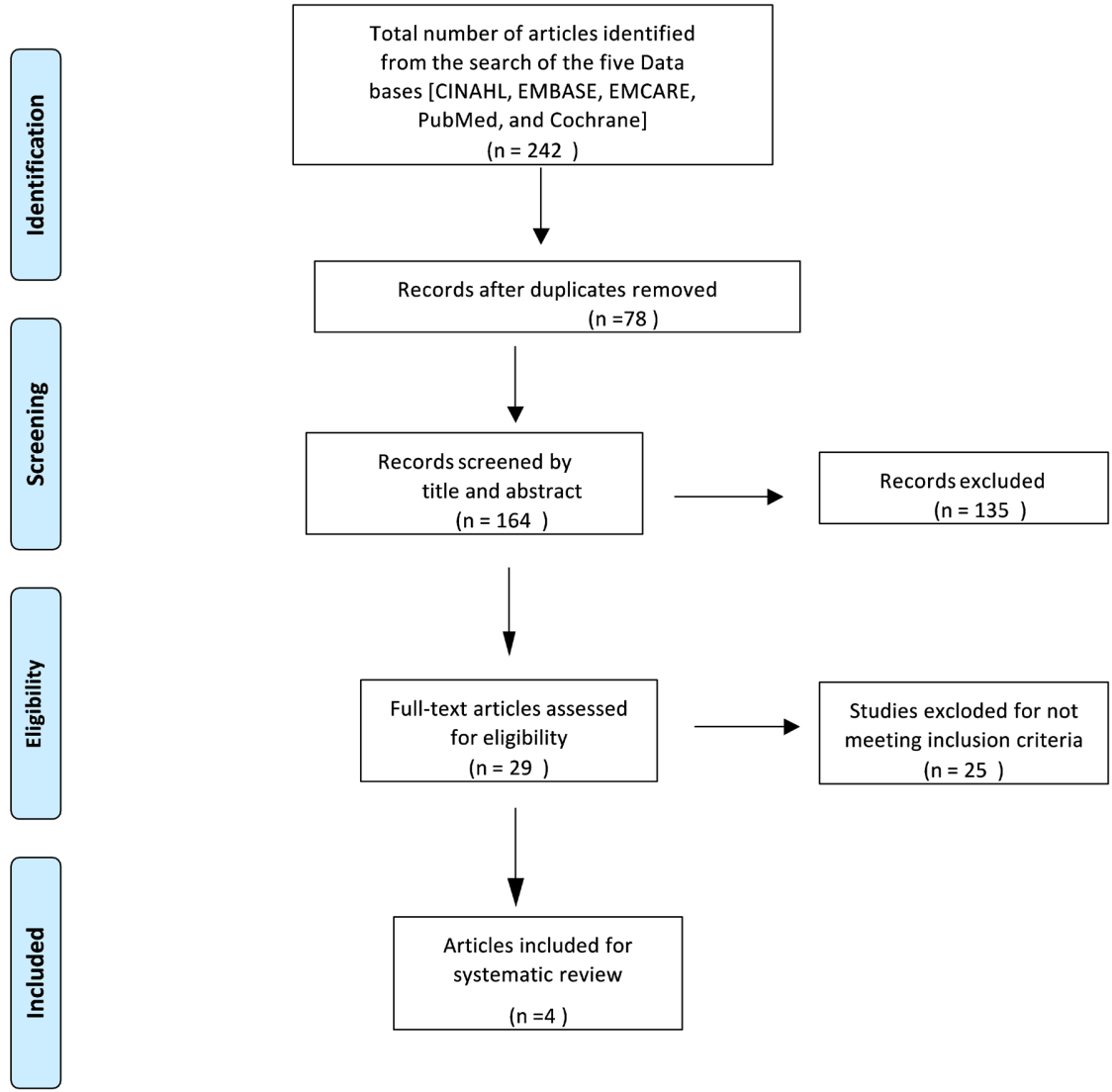

Figure 1. PRISMA flow diagram indicating the study selection process.

controlled trials was used to guide quality appraisal of the methods of the included studies (JBI, 2018). The characteristics assessed included randomisation, concealment, baseline similarities, blinding, follow up, methods of data analysis. The outcome of the critical appraisal of the included studies was presented in Table 2 .

In three of the included studies, there was randomisation of the participants to treatment groups [1] [9] [10]. Lubega [3] did not describe the participant's randomisation so an assessment of the quality of these methods was not possible.

All the studies concealed the allocation of the participants to treatment groups, using methods such as masking the allocation in opaque numbered study packets [10], labeling the syringes of ketamine and morphine with a sequence-generated code [3], sequentially numbered, sealed envelopes [1], and manila envelopes [9] to prevent patients and the researchers from knowing the treatment allocation.

The four studies ensured that the treatment groups were similar at baseline, comparing demographic characteristics such as; age, weight, gender, and race), pattern of injury, baseline pain scores, and vital signs [1] [3] [9] [10].

In all studies, the participants were blinded to their treatment [1] [3] [9] [10]. 
Only two studies blinded those who administered the treatment [1] [3]. Barcelos, [9] and Reynolds [10] did not report explicit strategies for blinding those who administered the treatment. Two of the studies blinded the outcome assessors [1] [10]. In the two remaining studies [3] [9], it is unclear whether the outcome assessors were blinded or not because there is no explicit explanation in the study articles.

The treatment groups were treated identically other than the interventions being investigated (opioid or ketamine) in all studies [1] [3] [9] [10]. The participants received similar treatment, such as vital sign assessment and provision of care [3], unblinded oral acetaminophen (maximum dose of $650 \mathrm{mg}$ ) or 10 $\mathrm{mg} / \mathrm{kg}$ ibuprofen (maximum dose of $600 \mathrm{mg}$ ) [10], pre-intervention treatment [9], and use of similar atomiser for medication delivery to patients [1]. All the included studies reported the follow up of the participants from screening, selection, through to completion of the [1] [3] [9] [10]. Thus, this ruled out the possibility of missing data as well and the withdrawal of patients from the study was documented. In all four included studies, the participants' data were analysed based on the group to which they were randomised [1] [3] [9] [10].

The outcomes were measured in the same and reliable way for treatment groups in each of all the included studies. Lubega [3] measured the outcomes for the treatment groups using numerical rating scale (NRS), while Reynolds [10] used the visual analogue scale (VAS) for children aged $11-17$ years and Face Pain Scale-Revised (FPS-R) for children 4 - 10 years to measure the outcomes in the treatment groups. Frey [1] also used the VAS pain scale and Michigan Sedation Scale (MSS) to assess pain in both treatment groups. Barcelos [9] also measured the outcomes similarly for the treatment groups, using a face pain scale. The pain scales used in the studies, such as VAS, NRS, and FPS-R were considered reliable, sensitive, and valid in terms of the provision of subjective and objective pain assessment in children.

The appropriateness of the statistical methods used was also assessed. Barcelos [9], Frey [1], and Lubega [3] used Student's t-test and Mann-Whitney U test to compare the outcome of the treatment groups. These methods were suitable because the test statistics (pain scores) followed not normally distributions. Frey [1], and Lubega [3] tested categorical variables using Chi-square $\left(\chi^{2}\right)$ or Fisher's exact test. Reynolds [10] also used chi-square to determine the statistical significance of the relationships between the treatment groups. Lastly, all the included studies had appropriate trial designs and used standard RCT designs. All the studies adhered to the randomisation of participants to treatment (case) and comparison (control) groups, concealment of treatment group allocation.

\subsubsection{Risk of Bias}

From the critical appraisal, various biases were identified. This included selection bias that occurs when eligible participants are not appropriately randomised to treatment groups, [11] as in the case of a study conducted by Lubega [3]. Secondly, performance bias was identified in two studies [9] [10] because of a lack 
of blinding those who were delivering treatment. Performance bias is attributed to differences in the treatment of the case and control groups due to knowledge and awareness of intervention allocations by the researcher and the participants [12]. Thirdly, information bias could have affected Barcelos [9] and Lubega [3] because of a lack of blinding the assessors of the outcome. Information bias occurs when the results are interpreted based on knowledge, reference test results, or other related information other than in practice [11]. Lack of blinding of assessors also increased the risk of detection bias, which results from the difference between the participants' treatment groups based on how the outcome was determined [12]. Lastly, excluded data bias could have affected a study by Lubega [3] because of the exclusion of other important data during analysis, such as the results of three participants in the ketamine group and one participant in morphine group from data analysis because they were discontinued from the study before outcomes. Excluded data bias occurs when some results are not included in the data analyses, because of uninterpretable tests or withdrawals of participants [9].

\subsection{Data Extraction and Characteristics of the Included Studies}

\subsubsection{Data Extractions Outcome}

Data were extracted from the selected studies was done using a table prescribed by JBI (2017). The key data extracted included authors of the study articles, year of publication, setting, the populations, interventions, and study results (see $\mathrm{Ta}$ ble 2) [5].

\subsubsection{Characteristics of the Included Studies}

The four studies included were double-blind randomised control trials (RCTs), [1] [3] [9] [10] (see Table 2). They were all published in the English language between 2012 to 2019). The studies were conducted in three different countries; Brazil [9], United States [1] [10], and Uganda [3]. Three studies focused on emergency departments, including paediatric emergency room and trauma centre [1] [9] [10]. The fourth study by Lubega [3], was conducted in a day-care sickle cell centre.

The four studies included paediatric (children) population aged between 3 18 years. For instance, Barcelos [10] included children aged 3 - 14 years, while Frey [1] recruited children aged $8-17$ years. Lubega [3] included children aged 7 - 18 years, while Reynolds [10] recruited children aged 4 - 17 years. The study sample sizes ranged from 25 to 240 . For instance, the largest study including a sample of 240 children [3], and the smallest study includes a sample of 25 [10]. Reynolds [11] and Frey [1] included samples of 91 and 90 respectively. All the study's had power calculations to established sample size, which was greater than $90 \%$ power. Three studies included children with trauma and injuries including dislocation, closed fractures [10], traumatic limb injury [1], and suspected isolated extremity fractures [11]. Lubega [3] recruited children with severe pain associated with an acute sickle cell crisis. 
Two studies investigated ketamine compared to morphine efficacy [3] [10], while the remaining two investigated ketamine compared to fentanyl efficacy [1] [11]. Barcelos [10] compared morphine $0.1 \mathrm{mg} / \mathrm{kg}$ administered intravenously, followed by increments of $0.05 \mathrm{mg} / \mathrm{kg}$ versus ketamine $2 \mathrm{mg} / \mathrm{kg}$ given intravenously up to a maximum dose of $70 \mathrm{mg}$. Lubega [3] investigated $1 \mathrm{mg} / \mathrm{kg}$ of intravenous ketamine versus intravenous morphine $0.1 \mathrm{mg} / \mathrm{kg}$. Frey [1] compared intranasal (IN) ketamine $(1.5 \mathrm{mg} / \mathrm{kg})$ or intranasal fentanyl $(2 \mu \mathrm{g} / \mathrm{kg})$, while Reynolds [11] compared intranasal sub-dissociative ketamine $(1 \mathrm{mg} / \mathrm{kg})$ to intranasal fentanyl $(1.5 \mathrm{mg} / \mathrm{kg})$.

\subsection{Key Findings of Each Included Study}

Meta-analysis was not conducted because of the heterogeneity of the included studies. For instance, the studies were performed in in different settings including the ED and sickle cell centre. The studies also used different pain scales to measure the outcome of the effectiveness of pain management with ketamine. Again, the studies involved different mode and route of delivery of the drugs (ketamine), such as intravenous and intranasal.The studies also used different data analysis methods and statistical tests.

The primary objective of this review was to compare the effectiveness of ketamine on the management of severe acute pain in children in the ED. The key findings are that the four included studies reported that ketamine had non-inferior analgesic effects when compared with opioids for the management of acute pain among children. Frey [1] reported that the mean visual analogue scale (VAS) reduction after 30 minutes of ketamine administration was $30.6 \mathrm{~mm}$ (95\% CI, 25.4 - 35.8) and $31.9 \mathrm{~mm}$ (95\% CI, 26.6 - 37.2) after fentanyl administration. In VAS, the patients were to score their pain intensity as no pain $(0-4 \mathrm{~mm})$, mild pain $(5-44 \mathrm{~mm})$, moderate pain $(45-74 \mathrm{~mm})$, as well as severe pain (75- 100 $\mathrm{mm}$ ) (Frey et al., 2018). Based on the observed confident interval, the finding is highly plausible and has a better estimate of pain management within the population. Additionally, the study reported that based on a one-sided test of group difference ketamine was non-inferior as the mean difference in pain reduction between groups was 1.3 (90\% CI, -6.2 to 8.7) [5]. Barcelos [10] measured pain using the Faces Pain Scale and found out that the median pain scores following the procedure were similar two for both ketamine and morphine. The median pain score following the procedure was 2 (mild pain) in both ketamine and morphine groups. In the Faces Pain Scale, the patient points at a face with a grimace of pain that represents his or her face. The faces are scaled from 1 - 10, with o denotes no pain and 10 means extreme pain. Lubega [3] found out that mean numerical rating scales (NRS) pain score for children randomised to the ketamine group was $66.4 \pm 29.9$, which was not statistically different to the mean pain scores $(61.3 \pm 28.7)$ for the morphine group (MD 5.5; 95\% CI -2.2 to -13.2). The numerical rating scale involves rating the patient's pain on a scale of $0-10$, whereby 0 is no pain and 10 is the extreme pain [3]. Moreover, the meantime to the maximum reduction in NRS pain scores for the ketamine group was 
60 minutes compared to 120 minutes for the morphine group (RR 1.2; 95\% CI 0.9 - 1.7). This was a small confidence interval which reinforced the reliability and accuracy of the findings. Reynolds [11] reported that the mean pain scale score decrease after 20 minutes was $44+36$ for the patients who received ketamine and $35+29$ for those who received fentanyl (mean difference $=9$ [95\% CI $=-4$ to 23]). This was a very wide confidence interval indicating the unreliability of the findings. From the findings, it can be deduced that ketamine was as superior as opioids in severe pain management in children. Hence, it could be used to effectively manage severe pain in children in the ED.

The second objective of this study was to describe some of the common side effects and adverse events associated with ketamine use compare to opioids in the management of acute pain in children in the emergency department and other severe and acute pain care settings. The reviewed studies found out that there was an increased risk of occurrence of various types and incidence of minor transient side effects associated with ketamine administration when compared with opioid administration. For instance, Frey [1] revealed that the risk of adverse events was higher in the ketamine group compared to the fentanyl group (relative risk, 2.5; 95\% CI, 1.5 - 4.0) [5]. This confidence interval was relatively narrow, which indicated considerable reliability of the findings. Barcelos [10] revealed that types of side effects, such as amnesia occurred in $92.3 \%$ of those who received ketamine and $83.3 \%$ for those who received morphine $(p=0.904)$. Lubega [3] identified that the patients in the ketamine group had 11.3 times the likelihood of developing transient non-threatening adverse effects compared to the morphine group (LDK 45(37.5\%) vs. MOR 4(3.3\%)). There were 2.2 times higher cumulative number of adverse events in the ketamine group than in the fentanyl group. The study also identified that some of the commonly reported side effects linked to ketamine included bad taste in the mouth ( $\mathrm{n}=37 ; 90.2 \%)$, dizziness ( $\mathrm{n}=30 ; 73.2 \%)$, and sleepiness $(\mathrm{n}=19 ; 46.3 \%)$. The commonest side effect reported in the fentanyl group included sleepiness $(15 ; 36.6 \%)$, bad taste in the mouth $(9 ; 22 \%)$, and itchy nose $(9 ; 22 \%)$ [12].

In summary, this systematic review included only four RCTs that met the inclusion criteria. This indicates that there is still a dearth of RCT studies that compare the effectiveness of ketamine and opioid in the management of acute and severe pain among children admitted to the emergency department. Concerning the first objective, the review has identified that ketamine has comparable effectiveness and efficacy to opioids in the management of pain in children admitted to the ED. On the second objective, ketamine use is associated with an increased incidence of occurrence of transient side effects and adverse events, which are minor and may not require clinical management or hinder the use of the drug.

\section{Discussion}

The main objective of this systematic review was to investigate the effectiveness of ketamine compared to opioids for managing acute pain experienced by child- 
ren in the Emergency Department (ED). The findings derived from the systematic review of the four RCTs revealed that that ketamine has non-inferior analgesic effects when used to manage acute and severe pain in children in the ED to opioids, such as morphine and fentanyl [1] [3] [10] [11]. The four reviewed studies agreed that ketamine was able to produce similar pain reductions outcomes as compared to opioids in children with acute and severe pain in the emergency department. Therefore, this suggests that ketamine can be used to provide analgesic outcomes comparable to opioids in managing severe pain in children in ED.

As a secondary outcome, this systematic review found that the use of ketamine in the management of severe pain in children in ED is associated with increased frequency of minor, transient, and non-life-threatening side effects compared to opioids, such as morphine and fentanyl [1] [3] [10] [11]. The four reviewed studies have linked ketamine with increased frequency of occurrence of minor side effects when used to treat severe pain in children in ED. According to the review, some of the identified common side effects linked to ketamine included dizziness and sleepiness, bad taste in the mouth, visual disturbances, itchy nose, sedation, and amnesia [1] [3] [10] [11]. Therefore, this suggests that the side effects produced by ketamine may not impede its use in managing severe pain in children in ED because they are temporary and non-life threatening.

\subsection{Support of the Evidence}

The findings generated by this systematic review are considered reliable for application in clinical practices. In terms of the level of evidence for effective clinical application, the evidence generated by this systematic review can be categorised as level 1A [11]. This is because a systematic review of RCTs often provides a reliable and dependable high level of evidence for clinical practice, which can guide decision making and a strong recommendation for clinical practice [13]. Despite including only four RCTs, this systematic review adhered to rigorous and transparent systematic review protocols prescribed by JBI protocol (2018), which has made the results consistent and reliable. The studies included were high-quality RCTs. The methodological quality appraisal of the included studies confirmed that the quality of the included studies was very high, increasing confidence in the results.

The findings regarding the effectiveness of ketamine in the management of severe pain in children in ED generated by this systematic review are consistent and corroborated by other existing systematic reviews on a similar topic, including those with broader inclusion criteria, populations, settings, and outcomes. For instance, a meta-analysis conducted by Lee and Lee [14] which included six trial studies with a total sample size of 438 patients reported that ketamine produced similar or superior analgesic outcomes compared to opioids. Although the study included studies that focused on adult patients aged 18 to 70 years. Another meta-analysis conducted by Sin [15], which included four RCTs 
with a total sample of 428 patients including both children and adults (5 - 70 years), identified that ketamine had a similar analgesic outcome as opioid. Both Lee and Lee [15] and Sin [15] were meta-analyses of RCTs, thus, the evidence they provided is of high level than this systematic review. They adhered to the meta-analysis procedures, which enhanced the consistency of the findings. The two meta-analyses included more studies which enhanced the confidence of the generalisability of the findings to a larger population of children with severe pain in ED. Therefore, the result generated by this systematic review is considered reliable and can be confidently used to guide clinical decisions concerning the use of ketamine to manage severe pain in children in ED.

The comparable efficacy of ketamine to opioids as identified by this systematic review has also corroborated by various RCTs studies. For instance, an RCT conducted by Mahsidfar [16] identified that ketamine provides a significant reduction of the average pain intensity comparable to morphine in adult patients. Majidinejad [17] also conducted a double-blind RCT to evaluate the effectiveness of ketamine alone in pain management in adult trauma patients and found out that ketamine and morphine had similar effects in alleviating pain. The two studies included relatively large sample sizes (300 and 126 trauma patients for Mahsidfar [16] and Majidinejad [17] respectively, which enhance confidence in the generated findings and possibility of generalisation of the findings. The effectiveness and safety of ketamine use in managing pain in children with acute and severe pain are attributed to its pharmacodynamic properties [18]. Ketamine works by binding to spinal $\mu$ receptors and increases the effectiveness of opioid-induced signaling [19]. Additionally, ketamine antagonises NMDA that preferentially acts at the postsynaptic receptors, which reduces hyperexcitability [19]. This prevents the postsynaptic neuronal hyperexcitability of pain and the occurrence of hyperalgesia, which is often associated with opioids [20] [21]. Thus, this makes it a suitable alternative analgesic agent to opioids in managing severe pain in children. The comparable analgesic effect of opioid and ketamine can be understood in terms of how the two drugs act different on pain receptors and the outcome of stimulating those receptors. For instance, opioids are considered to target limited number of specific opioid pain receptors [19]. On the other hand, ketamine targets multiple pain pathways simultaneously, which limits the possibility of hyperactivity through limited pain circuits [19] [22]. Thus, opioid is associated with dampening of acute pain transmission, but ketamine is considered to dampen pain response [19]. These effects are comparable, only that the limited pathway inhibited by opioid results to repeated stimulation that increases the possibility of downstream effects, which is associated with opioid dependence, addiction, and hyperalgesia [19]. These are avoided by ketamine, which inhibits wind-up and reduces central sensitisation, as well as chronic pain [19]. In this regard, ketamine appears to be a little safer and more effective in pain management compared to opioids. Concerning the second objective, there is widespread evidence that ketamine use in pain management in both children is associated with various minor temporary side effects, just as it has been identi- 
fied by this review [1] [10] [14] [15]. However, other studies have found that the side effect is not only in children but also in adults [14] [15]. A meta-analyses study by Lee and Lee [14] and Sin [15] identified that the ketamine produces only transient side effects that did not require clinical attention. However, Lee and [14] reported that the incidence of major cardiovascular events was much higher for opioids than ketamine Vadivelu [22] reported that ketamine produces minor effects such as sedation in both adult and paediatric patients. However, it does not result in major adverse dysphoric effects which are commonly observed in opioids [22]. An evidence appraisal conducted by Niesters, Martini, and Dahan [23] reported that despite being well-tolerated by both adult and paediatric patients, ketamine is still associated with the potential occurrence of psychedelic symptoms, such as hallucination, panic, and well as a hallucination. It also produces common minor side effects, such as nausea, vomiting, as well as somnolence [23]. This implies that ketamine is relatively safer compared to opioids, hence it can be safely used for pain management in children in ED.

In terms of treatment, the reviewed studies indicated most of the adverse events attributed to ketamine use are temporary and do not require treatment [1] [3] [10] [11]. However, some available evidence recommends that the side effects should be evaluated for possible [24]. For instance, Cohen [25] identified ketamine infusion to manage chronic pain in both children and adults is associated with few minor side effects that do not require treatment but, the patient should be monitored for potential health risk. Niesters, Martini, and Dahan [23] stated that there should be mandatory monitoring of all the patients receiving ketamine. Bell and Kalso [20] recommended that the side effects observed in ketamine use can be minimised or avoided by ensuring accurate dose and often using a low dose of ketamine. This is supported by Jonkman [18] who recommended that there should be personalised, cautious, as well as patient titration of ketamine infusion rate to produce minimal side effects. Therefore, it can be deduced that all patients receiving ketamine medication should be monitored for the potential occurrence of side effects and adverse events so that they can be treated in time to enhance patient safety. The side effects can also be minimised by using an appropriately low dose of ketamine based on the patient's characteristics.

\subsection{Clinical Implications and Recommendation}

In clinical practice, ketamine can be considered for use as an alternative to opioids in the management of acute and severe pain among children in ED. The evidence generated by this systematic review demonstrates that ketamine has similar non-superior analgesic outcomes as opioids. This systematic review has reinforced the widespread and growing view that ketamine is safe, effective, and a suitable alternative to opioids in the management of acute pain in children in the Emergency Department [1] [3] [10] [11]. Other literature has reported that the use of ketamine as an alternative to opioids is rapidly growing in clinical practice, especially in the management of acute and severe pain in the Emergen- 
cy Department [2] [26].

For instance, Jennings, Cameron, and Bernard [27] reported that there are widespread overall acceptance and prevalent implementation of ketamine in the management of acute and severe pain in children in ED. The increased use of ketamine as an alternative to opioids analgesics has been enhanced by various factors, such as the listing of ketamine by the American College of the Emergency Physicians (ACEP) as an appropriate alternative to opioids [28]. This has endorsed and promoted the credibility of ketamine use in the Emergency Department. However, Bell and Kalso [20] attribute the increased use of ketamine as an alternative to the opioid analgesic drug to its increased familiarity and popularity among emergency physicians and other health care professionals. Additionally, Schwenk [28] added that the increased decision to use ketamine in pain management in ED has been significantly influenced by increased multiple access to online medical education by healthcare professionals that are intending to publicise ketamine as an alternative to opioids. Therefore, based on the evidence generated by this systematic review, it can be confidently recommended that ketamine could be used as an alternative to opioids to effectively manage acute and severe pain in children in ED.

Ketamine can be used in cases where opioid analgesics are completely contraindicated or when an opioid analgesic is likely to produce an adverse reaction. For instance, ketamine can be the most appropriate alternative analgesic to patients who require potent analgesic but have opioid use problems and potential for prolonged use of opioids [29]. This is because prolonged use may cause addiction and dependence, and the patient who are using other medication conditions that are known to compromise opioid use [2] [30]. This is appropriate because evidence indicates that ketamine has the minimal potential of causing addictions compared to opioids [20] [31]. Lee and Lee [14] also argued that ketamine can minimise opioid dependence effects. Thus, it can be deduced that ketamine is a favourable analgesic among patients with opioid contraindications, such as the risk of opioid dependence. It is also agreeable that some uncommon medical conditions, such as chronic pulmonary disease and renal failure may contraindicate opioid uses because of possible severe respiratory distress and delayed elimination of opioids respective [21]. Thus, ketamine is the most suitable alternative in such cases.

\subsection{Gaps Remain}

One of the key gaps identified from the systematic review is that there is no clear evidence concerning the appropriate dose and route of administration of ketamine in the management of the severe pain in children. This systematic review did not determine the effective dose and route of administration that can be used to produce enough analgesic effects in pain management in children. The reviewed studies used different dosages as well as the route of administration of ketamine during treatment, such as intravenous, intranasal, and intramuscular. Thus, it would be difficult to draw conclusions about the most appropriate do- 
sage and suitable route of administration when using ketamine to manage acute and severe pain in children. Based on this gap, it is advisable for the ED health professionals to determine the appropriate dose for ketamine based on the child's characteristics, such as weight, age, and disease condition.

\subsection{Strengths and Limitations}

The key strength of this systematic review is the use of the JBI systematic review protocol, which reinforced the rigor of the process. This makes the results reliable and dependable. Secondly, the use of three reviewers during the data search, extractions, and quality assessment, which enhanced the consistency of the process and the findings. Using strict inclusion and exclusion criteria, this systematic review restricted numerous potential confounding variables by establishing strict inclusion criteria in terms of populations, interventions, and outcomes [5].

One of the key weaknesses of the systematic review is the inclusion of a few studies that addressed the question. Secondly, their heterogeneity of the included studies also prevented meta-analysis and therefore a result with more statistical power to answer the question. Additionally, there was no performance of sensitivity analysis and meta-regression to determine heterogeneity since only a small number of studies were included. There was also no calculation of reliability, particularly with the studies' eligibility and selection procedure, since the three reviewers were in full agreement. There was no performance of the risk of publication bias.

\section{Conclusion}

Ketamine is a suitable alternative for opioid analgesics for the management of acute and severe pain in children in ED. The evidence generated by this study is that ketamine is non-inferior to opioids (morphine and fentanyl) in controlling acute pain in children. The study revealed that although ketamine use is associated with increased risk of occurrence of side effects and adverse effects, they are transient, non-life-threatening and may not need serious clinical intervention. Therefore, this systematic review supports the possibility of the use of ketamine as an alternative to opioids in the management of severe pain. This includes the use of ketamine in patients with opioid contraindication or in cases, where opioid is likely to cause serious side effects or adverse outcome. However, in clinical practice, health ketamine should not be considered as a complete replacement for opioid analgesics.

\section{Conflicts of Interest}

The author declares no conflicts of interest regarding the publication of this paper.

\section{References}

[1] Frey, T., Florin, T., Caruso, M., Zhang, N., Zhang, Y. and Mittiga, M. (2019) Effect 
of Intranasal Ketamine vs Fentanyl on Pain Reduction for Extremity Injuries in Children: The PRIME Randomised Clinical Trial. JAMA Paediatric, 173, 140-146. https://doi.org/10.1001/jamapediatrics.2018.4582

[2] Karlow, N., Schlaepfer, C., Stoll, C., Doering, M., Carpenter, C., Colditz, G., Motov, S., Miller, J. and Schwartz, E. (2018) A Systematic Review and Meta-Analysis of Ketamine as an Alternative to Opioids for Acute Pain in the Emergency Department. Academic Emergency Medicine, 25, 1086-1097.

[3] Lubega, F.A. DeSilva, M., Munube, D., Nkwine, R., Tumukunde, J., Agaba, P., Nabukenya, M., Luggya, T and Bulamba, F. (2018) Low Dose Ketamine versus Morphine for Acute Severe Vaso Occlusive Pain in Children: A Randomised Controlled Trial. Scandinavian Journal of Pain, 18, 19-27.

https://doi.org/10.1515/sjpain-2017-0140

[4] Rubinstein, O., Barkan, S., Breitbart, R., Berkovitch, S., Toledano, M., Weiser, G., Karadi, N., Nassi, A. and Kozer, E. (2016) Efficacy of oral Ketamine Compared to Midazolam for Sedation of Children Undergoing Laceration Repair: A Double-Blind, Randomized, Controlled Trial. Medicine, 95, e3984. https://doi.org/10.1097/MD.0000000000003984

[5] Joanna Briggs Institute (2017) The Joanna Briggs Institute Reviewers' Manual 2017. Methodology for JBI Scoping Reviews. The Joanna Briggs Institute, Adelaide.

[6] Sriganesh, K., Shanthanna, H. and Busse, J. (2016) A Brief Overview of Systematic Reviews and Meta-Analyses. Indian Journal of Anaesthesia, 60, 689-694.

[7] Ahn, E. and Kang, H. (2018) Introduction to Systematic Review and Meta-Analysis. Korean Journal of Anesthesiology, 71, 103-112.

https://doi.org/10.4097/kjae.2018.71.2.103

[8] Miro, J., Castarlenas, E. and Huguet, A. (2009) Evidence for the Use of a Numerical Rating Scale to Assess the Intensity of Pediatric Pain. European Journal of Pain, 13, 1089-1095. https://doi.org/10.1016/j.ejpain.2009.07.002

[9] Barcelos, A., Garcia, P., Portela, J., Piva, J., Garcia, T. and Santana, J. (2015) Comparison of Two Analgesia Protocols for the Treatment of Pediatric Orthopedic Emergencies. Revista da Associação Médica Brasileira, 61, 362-367. https://doi.org/10.1590/1806-9282.61.04.362

[10] Reynolds, S., Bryant, K., Studnek, J., Hoog, M., Dunn, C., Templin, M., Moore, C., Young, J., Walker, K. and Runyon, M. (2017) Randomized Controlled Feasibility Trial of Intranasal Ketamine Compared to Intranasal Fentanyl for Analgesia in Children with Suspected Extremity Fractures. Academic Emergency Medicine, 24, 1430-1440. https://doi.org/10.1111/acem.13313

[11] Joanna Briggs Institute (2019) JBI Reviewer's Manual: 9.2.5 Assessment of Methodology Quality.

[12] Mansournia, M.A., Higgins, J.P.T., Sterne, J.A.C. and Hernán, M.A. (2017) Biases in Randomized Trials: A Conversation between Trialists and Epidemiologists. Epidemiology, 28, 54-59. https://doi.org/10.1097/EDE.0000000000000564

[13] Cheung, M. (2015) Meta-Analysis: A Structural Equation Modelling Approach. John Wiley \& Sons, New York. https://doi.org/10.1002/9781118957813

[14] Lee, E. and Lee, J., (2016) The Effects of Low-Dose Ketamine on Acute Pain in an Emergency Setting: A Systematic Review and Meta-Analysis. PLOS ONE, 11, e0165461. https://doi.org/10.1371/journal.pone.0165461

[15] Sin, B., Ternas, T. and Motov, S. (2015) The Use of Sub Dissociative-Dose Ketamine for Acute Pain in the Emergency Department. Academic Emergency Medicine, 22, 251-257. https://doi.org/10.1111/acem.12604 
[16] Mahshidfar, B., Mofidi, M., Fattahi, M., Farsi, D., Moghadam, P., Abbasi, S. and Rezai, M. (2017) Acute Pain Management in the Emergency Department, Low Dose Ketamine versus Morphine, A Randomized Clinical Trial. Anesthesiology and Pain Medication, 7, e60561.

[17] Majidinejad, S., Esmailian, M. and Emadi, M. (2014) Comparison of Intravenous Ketamine with Morphine in Pain Relief of Long Bones Fractures: A Double-Blind Randomized Clinical Trial. Emergency, 2, 77-80.

[18] Jonkaman, K., Dahan, A., Donk, T., Niesters, M. and Velzen, M. (2017) Ketamine for Pain. F1000Research, 6, 12-19. https://doi.org/10.12688/f1000research.11372.1

[19] Gao, M., Rejaei, D. and Liu, H. (2016) Ketamine Use in Current Clinical Practice. Acta Pharmacologica Sinica, 37, 865-872. https://doi.org/10.1038/aps.2016.5

[20] Bell, F. and Kalso, E. (2018) Ketamine for Pain Management. Pain Reports, 3, 674-682. https://doi.org/10.1097/PR9.0000000000000674

[21] Matson, K., Johnson, P., Tran, V., Horton, E. and Sterner-Allison, J. (2019) Opioid Use in Children. The Journal of Pediatric Pharmacology and Therapeutics, 24, 72-75. https://doi.org/10.5863/1551-6776-24.1.72

[22] Vadivelu, N., Schermer, E., Kodumudi, V., Belani, K., Urman, R. and Kaye, A. (2016) A Systematic Review and Meta-analysis of Ketamine as an Alternative to Opioids for Acute Pain in the Emergency Department. Journal of Anaesthesiology Clinical Pharmacology, 32, 298-306.

[23] Niesters, M., Martini, C. and Dahan, A. (2014) Ketamine for Chronic Pain: Risks and Benefits. British Journal of Clinical Pharmacology, 77, 357-367.

https://doi.org/10.1111/bcp.12094

[24] Kurdi, M.S., Theerth, K.A. and Deva, R.S. (2014) Ketamine: Current Applications in Anesthesia, Pain, and Critical Care. Anesthesia: Essays and Researches, 8, 283-290. https://doi.org/10.4103/0259-1162.143110

[25] Cohen, S., Bhatia, A., Buvanendran, A., Schwenk, E., Wasan, Hurley, R., Viscusi, E., Narouze, S., Davis, F., Ritchie, E., Lubenow, T. and Hooten, W. (2018) Consensus Guidelines on the Use of Intravenous Ketamine Infusions for Chronic Pain from the American Society of Regional Anesthesia and Pain Medicine, the American Academy of Pain Medicine, and the American Society of Anesthesiologists. Regional Anesthesia and Pain Medicine, 43, 521-546. https://doi.org/10.1097/AAP.0000000000000808

[26] Kapural, L., Kapural, M., Bensitel, T. and Sessler, D. (2010) The Opioid-Sparing Effect of Intravenous Outpatient Ketamine Infusions Appears Short-Lived in Chronic-Pain Patients with High Opioid Requirements. Pain Physician, 13, 389-394. https://doi.org/10.36076/ppj.2010/13/389

[27] Jennings, P., Cameron, P. and Bernard, S. (2011) Ketamine as an Analgesic in the Pre-Hospital Setting: A Systematic Review. Acta Anaesthesiologica Scandinavica, 55, 638-643. https://doi.org/10.1111/j.1399-6576.2011.02446.x

[28] Schwenk, E., Viscusi, E., Buvanendran, A., Hurley, R., Wasan, A., Narouze, S., Bhatia, A., Davis, F., Hooten, W. and Cohen, S. (2018) Consensus Guidelines on the Use of Intravenous Ketamine Infusion for Acute Pain Management from the American Society of Regional Anesthesia and Pain Medicine, the American Academy of Pain Medicine, and the American Society of Anesthesiologist. Regional Anesthesia and Pain Medicine, 43, 456-466.

https://doi.org/10.1097/AAP.0000000000000806

[29] BinKharfi, M. and AlSagre, A. (2019) BET 2: Safety and Efficacy of Low-Dose Ketamine versus Opioids for Acute Pain Management in the ED. Emergency Medical 
Journal, 36, 128-129.

[30] Jensen, T. and Finnerup, N. (2014) Allodynia and Hyperalgesia in Neuropathic Pain: Clinical Manifestations and Mechanisms. The Lancet Neurology, 13, 924-935. https://doi.org/10.1016/S1474-4422(14)70102-4

[31] Maher, D., Zhang, Y., Ahmed, S., Doshi, T., Malarick, C., Stabach, K., Mao, J. and Chen, L. (2017) Chronic Opioid Therapy Modifies QST Changes after Ketamine Infusion in Chronic Pain Patients. Journal of Pain, 18, 1468-1475.

https://doi.org/10.1016/j.jpain.2017.07.008 